\title{
Variation of cutting forces in machining of f.c.c. single crystals
}

\author{
S. Abolfazl Zahedi ${ }^{1}$, Anish Roy ${ }^{2}$, Vadim V. Silberschmidt ${ }^{3}$
}

Wolfson School of Mechanical and Manufacturing Engineering, Loughborough University, LE11 3TU UK

${ }^{1}$ S.Zahedi@lboro.ac.uk, +44 (0)141 5345565,

${ }^{2}$ A.Roy3 @lboro.ac.uk, +44 (0)1509 227504,

${ }^{3}$ V.Silberschmidt@lboro.ac.uk,+44 (0)1509 227637

\begin{abstract}
In this study, micro-machining of f.c.c single-crystal materials was investigated based on a hybrid modelling approach combining smoothed particle hydrodynamics and continuum finite element analysis. The numerical modelling was implemented in a commercial software ABAQUS/Explicit by employing a user-defined subroutine VUMAT for a crystal-plasticity formulation to gain insight into the underlying mechanisms that drive a plastic response of materials in highdeformation processes. The numerical studies demonstrate that cutting-force variations in different cutting directions are similar for different f.c.c. crystals even though the magnitudes of the cutting forces are different
\end{abstract}

Keyword: Single crystal, f.c.c., Shear strain, Cutting-force variation, SPH

\section{Introduction}

In recent years mechanical micro-machining has received much attention in the manufacture of industrial small-size components with complex geometries, especially for applications in aerospace, biomedical and automotive industries and microelectronics [1-2]. A growing demand from various applications to reduce levels of defects in ultra-precision metal cutting requires a fundamental understanding of machining mechanisms at the micro scale. These applications basically involve the machining of single-crystal metals or an aggregate of single crystals (polycrystalline material) where each crystal may be oriented in a different crystallographic direction in comparison to its neighbours. Apparently, machining of polycrystalline materials in the micro-scale is inherently different from machining single crystals. From a fundamental point of view, it is of interest to investigate the response of machining single crystal materials in different crystallographic orientations and directions of cutting. This will ultimately 
indicate the consequence for machining polycrystalline aggregates, albeit ignoring the effect of grain boundaries [3-4].

Analysis of any machining technique by means of extensive experimentation is an expensive and time-consuming process. In addition, complexity of the underlying physics of single-crystal deformation severely affects the outcomes of machining. As a result, there has been a significant thrust in the development of analytical and numerical computation methods for characterisation of micro-machining processes. For instance, Sato et al. [5] used the Schmid factor to predict active slip systems during the machining process in their model. They assumed one slip system active continuously in each orientation setups. Micro-plasticity modelling of machining proposed by Lee et al. [6-7] is another limited research available in the literature analysing a mechanism of single-crystal machining. Shirakashi et al. [8] and Kota and Ozdoganlar [9] used the Bishop and Hill's crystal plasticity model to predict shear angles and specific energies for f.c.c. single crystals.

The above techniques used to assess forces and stresses in machining process of single crystals are usually limited to one active slip system at each incremental deformation. Deformation processes in real-life machining are more complex and require the use of a comprehensive modelling framework in analysing the cutting forces and stresses involved. Some researchers used molecular dynamic (MD) simulation to study the chip-removal mechanism [10-12] but his approach requires significant computational power in order to model a cutting process in physically meaningful volumes. Therefore, many MD simulations were applied in two-dimensional formulations for a small workpiece with unrealistically high cutting speeds.

Selecting materials for single-crystal machining studies, mainly copper and aluminium have been preferred in the literature [3-12]. In this paper a welldeveloped computational FE/SPH model was applied for these two monocrystalline materials to fully understanding the variation of cutting forces. Copper and aluminium have both f.c.c. structures. The plastic deformation was a result of resolved shear stress on 12 possible slip systems, with the Schmid factor determining the slip-system activation. The crystal-plasticity formulation presented in the next section was implemented as a VUMAT subroutine for employment in ABAQUS/Explicit together with the SPH (smoothed particle 
hydrodynamics) technique to predict the deformations, stresses, plastic strain distribution in the f.c.c. crystalline structure of the workpiece materials. This allows the effects of crystallinity parameters on cutting-force variations to be investigated thoroughly.

\section{Crystal plasticity}

A crystal-plasticity framework has been widely used in predicting the mechanical behaviour of, and texture evolution in, f.c.c. materials. In the crystal-plasticity formulation the stress rate $\dot{\boldsymbol{\sigma}}$ is related to the elastic strain rate $\boldsymbol{L}^{\mathrm{e}}$ as

$$
\dot{\sigma}=C L^{\mathrm{e}}=C\left(L-L^{P}\right),
$$

where $\boldsymbol{C}$ is the fourth-order elasticity tensor and $\boldsymbol{L}$ and $\boldsymbol{L}^{\boldsymbol{P}}$ are the total strain rate and plastic strain rate, respectively. The f.c.c. metals have cubic symmetry; the elastic moduli for such crystals are particularly simple, and can be parameterized by only 3 material constants: $\boldsymbol{C}_{11}, \boldsymbol{C}_{12}$ and $\boldsymbol{C}_{44}$. The following matrix expresses the elastic moduli of such materials:

$$
\boldsymbol{C}=\left[\begin{array}{cccccc}
\boldsymbol{C}_{11} & \boldsymbol{C}_{12} & \boldsymbol{C}_{12} & 0 & 0 & 0 \\
\boldsymbol{C}_{12} & \boldsymbol{C}_{11} \boldsymbol{C}_{12} & 0 & 0 & 0 \\
\boldsymbol{C}_{12} & \boldsymbol{C}_{12} & \boldsymbol{C}_{11} & 0 & 0 & 0 \\
0 & 0 & 0 & \boldsymbol{C}_{44} & 0 & 0 \\
0 & 0 & 0 & 0 & \boldsymbol{C}_{44} & 0 \\
0 & 0 & 0 & 0 & 0 & \boldsymbol{C}_{44}
\end{array}\right]
$$

The plastic deformation $\boldsymbol{F}^{\mathrm{p}}$ represents material's plastic shear and corresponds to the amount of deformation that remains in the crystal after the load removal. According to the flow rule:

$$
\dot{\boldsymbol{F}}^{\mathrm{p}}=\boldsymbol{L}^{\mathrm{P}} \boldsymbol{F}^{\mathrm{P}}
$$

The plastic strain rate is assumed to be the sum of the shear strain rates $\dot{\gamma}^{\alpha}$ over the number of considered slip systems. Therefore,

$$
\boldsymbol{L}^{\mathrm{P}}=\sum_{\alpha=1}^{\mathrm{N}} \mu_{\mathrm{ij}}^{\alpha} \dot{\gamma}^{\alpha},
$$

with $\mu_{\mathrm{ij}}^{\alpha}$ is the Schmid tensor that is equal to a dyadic product of the slip direction $s_{\mathrm{i}}^{\alpha}$ and the slip plane normal $n_{\mathrm{j}}^{\alpha}$ : 


$$
\mu_{\mathrm{ij}}^{\alpha}=s_{\mathrm{i}}^{\alpha} \times n_{\mathrm{j}}^{\alpha}
$$

In Eqs. 4 and 5 the superscript $\alpha$ specifies the slip system and $\mathrm{N}$ is the total number of available slip systems.

The shear strain rate $\dot{\gamma}^{\alpha}$ of the $\alpha$ th slip system in a rate-dependent crystalline solid is determined by a visco-plastic flow rule as

$$
\dot{\gamma}^{\alpha}=\dot{\gamma}_{0} f\left(\frac{\tau^{\alpha}}{g^{\alpha}}\right)
$$

where the constant $\dot{\gamma}_{0}$ is the reference strain rate on the slip system $\alpha, g^{\alpha}$ is the variable, which describes the current strength of that slip system at the current time, $\tau^{\alpha}$ is the shear stress on slip system $\alpha$, and the non-dimensional function $f$ describes the dependence of strain rate on stress. The simplest flow rule is a viscoplastic power-law expression proposed by Hutchinson [13] to describe $\dot{\gamma}^{\alpha}$ in the following form:

$$
\dot{\gamma}^{\alpha}=\dot{\gamma}_{0} \operatorname{sgn}\left(\tau^{\alpha}\right)\left|\frac{\tau^{\alpha}}{g^{\alpha}}\right|^{\mathrm{n}}
$$

where $\mathrm{n}$ is the material's rate sensitivity and $\operatorname{sgn}(*)$ is the signum function of $*$. It is worth mentioning that the reference strain-rate $\dot{\gamma}_{0}$ in this equation is assumed to be $10^{-4} 1 / \mathrm{s}$. The strength of material $g^{\alpha}$ is equal to a sum of the critical resolved shear stress (CRSS) and the evolved slip-resistance due to strain hardening:

$$
g^{\alpha}=\left.g^{\alpha}\right|_{t=0}+\Delta g^{\alpha}
$$

where

$$
\Delta g^{\alpha}=\sum_{\beta=1}^{N} h_{\alpha \beta} \Delta \gamma^{\beta}, \text { CRSS }=\left.g^{\alpha}\right|_{t=0}
$$

The hardening moduli $h_{\alpha \beta}$ in Eq. (9) are evaluated using the hardening model proposed by Peirce et al. [14] as follows:

$$
\begin{gathered}
h_{\alpha \alpha}=h(\gamma)=h_{0} \operatorname{sech}^{2}\left|\frac{h_{0} \gamma}{\left.g_{\mathrm{T}}^{\alpha}\right|_{\text {sat }}-\left.g_{\mathrm{T}}^{\alpha}\right|_{t=0}}\right| \\
h_{\alpha \beta}=q h_{\alpha \alpha}(\alpha \neq \beta)
\end{gathered}
$$


where $h_{0}$ is the initial hardening parameter, $q$ is the latent hardening ratio and assumed to be $1, \gamma$ is the Taylor cumulative shear strain on all slip systems and $\left.g_{\mathrm{T}}^{\alpha}\right|_{t=0}$ and $\left.g_{\mathrm{T}}^{\alpha}\right|_{\text {sat }}$ are the shear stresses at the onset of yield and the saturation of hardening, respectively. Therefore, the shear strain is equal to

$$
\gamma=\sum_{\alpha} \int_{0}^{t}\left|\dot{\gamma}^{\alpha}\right| d t
$$

The user-defined material subroutine VUMAT, initially developed by Huang [15], modified by Kysar [16] and Zahedi [17-18] further developed by Demiral [19] was used to implement this single-crystal plasticity formulation. The eight parameters - $\tau_{s}, \tau_{0}, h_{0}, C_{11}, C_{22}, C_{44}, \dot{\gamma}_{0}, n$ - were considered as input material data. Table 1 lists the material parameters used in present simulations for copper and aluminium.

Table 1 Material parameters of single-crystal copper [20] and aluminum [21]

\begin{tabular}{|ll|}
\hline Copper & Aluminium \\
\hline $\boldsymbol{C}_{\mathbf{1 1}}=168 \mathrm{GPa}$ & $\boldsymbol{C}_{\mathbf{1 1}}=108.2 \mathrm{GPa}$ \\
\hline $\boldsymbol{C}_{\mathbf{1 2}}=121.4 \mathrm{GPa}$ & $\boldsymbol{C}_{\mathbf{1 2}}=\mathbf{6 1 . 3 \mathrm { GPa }}$ \\
\hline $\boldsymbol{C}_{\mathbf{4 4}}=\mathbf{7 5 . 4 \mathrm { GPa }}$ & $\boldsymbol{C}_{\mathbf{4 4}}=\mathbf{2 8 . 5 \mathrm { GPa }}$ \\
\hline$\dot{\boldsymbol{\gamma}}_{\mathbf{0}}=\mathbf{0 . 0 0 1 \mathrm { s } ^ { - 1 }}$ & $\dot{\boldsymbol{\gamma}}_{\mathbf{0}}=\mathbf{0 . 0 0 1 \mathrm { s } ^ { - 1 }}$ \\
\hline $\boldsymbol{n}=\mathbf{2 0}$ & $\boldsymbol{n}=\mathbf{2 0}$ \\
\hline $\boldsymbol{h}_{\mathbf{0}}=\mathbf{1 8 0 \mathrm { MPa }}$ & $\boldsymbol{h}_{\mathbf{0}}=\mathbf{3 . 4 \mathrm { MPa }}$ \\
\hline$\tau_{\boldsymbol{s}}=148 \mathrm{MPa}$ & $\tau_{\boldsymbol{s}}=\mathbf{4 8} \mathrm{MPa}$ \\
\hline$\tau_{\mathbf{0}}=\mathbf{1 6} \mathrm{MPa}$ & $\tau_{\mathbf{0}}=\mathbf{2 9} \mathrm{MPa}$ \\
\hline
\end{tabular}

The data for mono-crystalline materials in Table 1 show that apart form $\dot{\gamma}_{0}$ and $n$, which are the reference strain rate and material's rate sensitivity, respectively, the elastic parameters $C_{11}, C_{22}, C_{44}$ and the plastic parameters $\tau_{s}, \tau_{0}, h_{0}$ are different for copper and aluminium. In the following section the machining model is presented followed by discussion of deformation mechanisms.

\section{Machining model}

The basic mechanism of chip formation can be understood with the use of a simple process of orthogonal cutting. Thus, a 3D workpiece with dimensions of $500 \mu \mathrm{m} \times 500 \mu \mathrm{m} \times 50 \mu \mathrm{m}$ was selected as an appropriate representation of a 
crystalline continuum in simulations. The workpiece was divided into two regions; one representing the SPH domain $(200 \mu \mathrm{m} \times 200 \mu \mathrm{m} \times 50 \mu \mathrm{m})$ and the remaining part being a continuum FE domain (Figure 1). By coupling the SPH with FEM a large fraction of the model not in the immediate vicinity of the process zone is assumed to be the continuum FE domain. As a result, the computational time reduced significantly. Note that the accuracy of the FE/SPH model was deliberated in our previous research study [3].

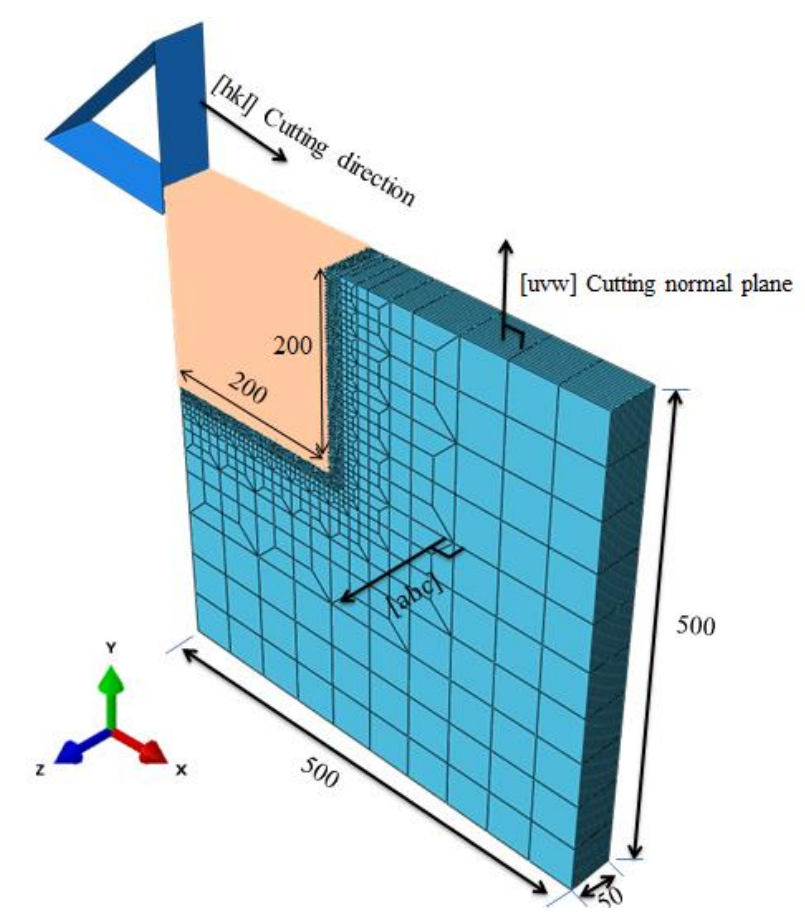

Figure 1: Orthogonal machining model (dimension in $\mu \mathrm{m}$ )

In our simulations, the appropriate crystal orientations - [010], [101] and [111] were selected as the normal for the cuttings plane, and the cutting process was carried out in four cutting directions: $0^{\circ}, 30^{\circ}, 60^{\circ}$ and $90^{\circ}$. The corresponding cutting directions and orientations are shown in Figure 1 (together with the notation used), and four orthonormal directions in each case calculated and listed in Table 2.

Table 2 Cutting orientation set (see Figure 1)

\begin{tabular}{|c|c|c|c|c|c|c|c|c|}
\hline \multicolumn{3}{|c|}{$0^{\circ}$} & \multicolumn{2}{|c|}{$30^{\circ}$} & \multicolumn{2}{|c|}{$60^{\circ}$} & \multicolumn{2}{|c|}{$90^{\circ}$} \\
\hline [uvw] & [abc] & [hkl] & [abc] & [hkl] & [abc] & [hkl] & [abc] & [hkl] \\
\hline [010] & [100] & [001] & {$\left[\begin{array}{lll}\sqrt{3} & 0 & \overline{1}\end{array}\right]$} & {$\left[\begin{array}{lll}1 & 0 & \sqrt{3}\end{array}\right]$} & {$\left[\begin{array}{lll}1 & 0 & \overline{\sqrt{3}}\end{array}\right]$} & {$\left[\begin{array}{lll}\sqrt{3} & 0 & 1\end{array}\right]$} & {$[00 \overline{1}]$} & [100] \\
\hline
\end{tabular}




\begin{tabular}{|c|c|c|c|c|c|c|c|c|}
\hline [101] & {$[\overline{1} 01]$} & [010] & {$[\overline{3} \sqrt{6} 3]$} & {$\left[\begin{array}{lll}1 & \sqrt{6} & \overline{1}\end{array}\right]$} & {$[\overline{\sqrt{2}} 2 \sqrt{3} \sqrt{2}]$} & {$[\sqrt{6} 2 \overline{\sqrt{6}}]$} & [010] & 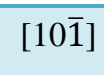 \\
\hline$[11$ & {$\left[\begin{array}{ll}\overline{1} & 01\end{array}\right]$} & $2 \overline{1}]$ & {$\left[\begin{array}{lll}\overline{1} & \overline{1} & 2\end{array}\right]$} & {$\left[\begin{array}{lll}\overline{1} & 1 & 0\end{array}\right]$} & {$\left[\begin{array}{lll}0 & \overline{1} & 1\end{array}\right]$} & {$\left[\begin{array}{lll}\overline{1} & 2 & \overline{1}\end{array}\right]$} & {$\left[\begin{array}{lll}\overline{1} & 2 & \overline{1}\end{array}\right]$} & {$\left[\begin{array}{ll}10 & \overline{1}\end{array}\right]$} \\
\hline
\end{tabular}

\section{Shear strain distribution}

The deceptively simple orthogonal single-crystal machining process actually involves complex phenomena crossing the fields of elasticity and plasticity. When the cutting tool advanced towards the workpiece, the latter started to deform. In particular, when the primary shear stress generated due to tool penetration is larger than the critical shear stress of single crystal material, plastic slip is initiated in the lattices to release the strain energy. In the presented crystal-plasticity theory the material flow rate on a slip system was represented in a continuum sense as a plastic shear strain $\gamma$ (Eq. 11). A change in a shear-strain direction and magnitude after cutting-tool penetration was calculated and updated in the model based on the VUMAT subroutine. In general, the active slip systems for the f.c.c. singlecrystal structure consist of $\{111\}$ slip planes and $<110>$ slip directions. There are 12 slip systems in total for f.c.c. structure which can be active or not at the same time. The activation of each slip system is determined by the value of Schmid factor. From the Schmid's law, it is apparent that the primary slip system will be the system with the greatest Schmid factor in terms of its magnitude. Figure 2 shows the distribution of shear strain over three slip systems after the cutting length reached $50 \mu \mathrm{m}$. These three slip systems were the most active ones, whereas the contributions of other systems were comparatively small. It is to be noted that the Schmidt factors for these three slip systems were equal to - $0.4082,0$ and 0 initially. This analysis was set at (010)[100] orientation setup with $10 \mu \mathrm{m}$ cutting depth, cutting tool speed equal to $1.3 \mathrm{~m} / \mathrm{s}$ and friction coefficient equal to 0.12 .

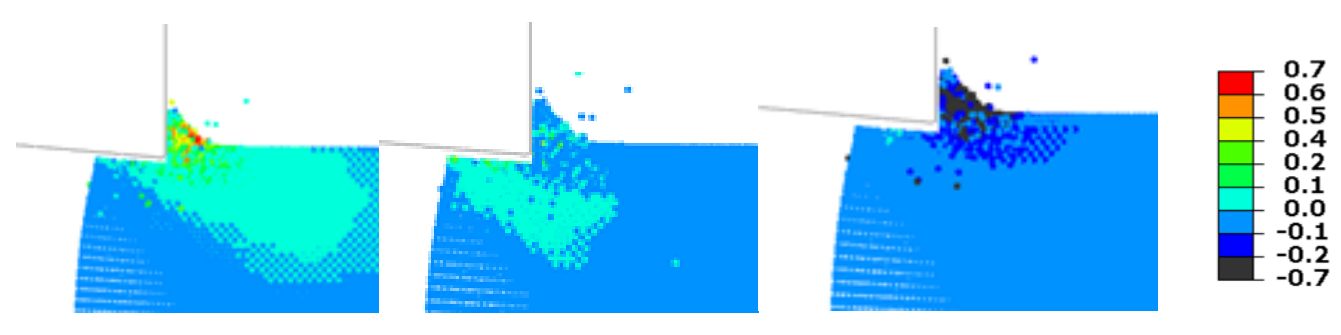


(1111) [101]

$(1 \overline{1} 1)[011]$

$(11 \overline{1})[101]$

Figure 2: Plastic shear strain $\gamma$ : distribution over three slip systems

\section{Effect of material property}

To study the sensitivity of cutting force to variation in material properties, the developed three-dimensional FE/SPH model was used to analyse cutting of single crystals of aluminium and copper. Figure 3 shows the cutting force variation for chosen cutting configurations (see Table 2) in aluminium and copper. It demonstrates a noticeable difference in the cutting-force magnitude with a similar trend in cutting-force variation across different slip planes and cutting directions for the two materials. This emanates from the underlying difference in dislocation activity in the two materials reflected in their elastic and plastic material properties; however, the similarity in the trend indicates that kinematics of deformation, which depend on the arrangement of the slip systems (identical for all f.c.c. metals) plays a major role in determining the nature of the cutting force.

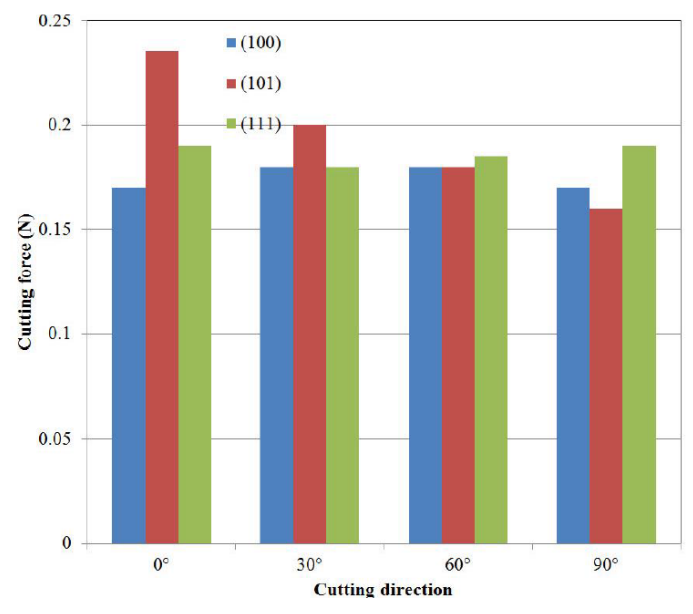

(a)

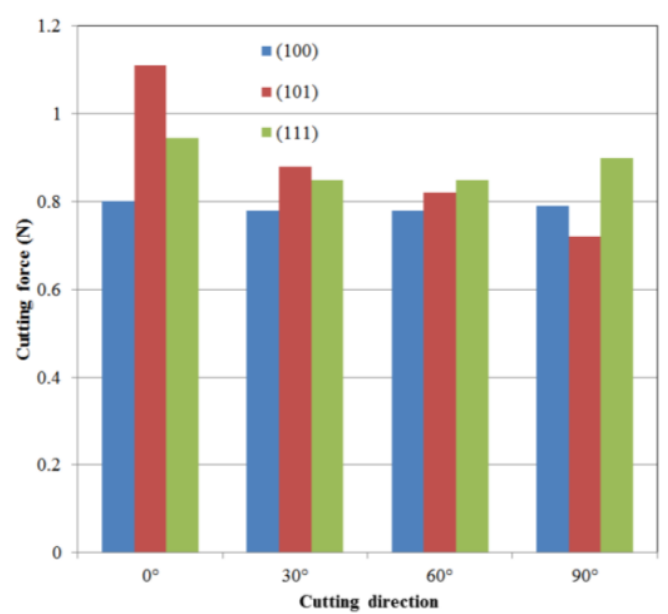

(b)

Figure 3 Typical variation of cutting force for various cutting directions in single crystals of aluminum (a) and copper (b)

Figure 4 also demonstrates that the total slip that occurs in all active slip systems for a given state of deformation is nearly the same for any f.c.c. structure. For example, when the aluminium or copper crystal were oriented in the (100) plane and cut in the [100] direction, the chip formation appears 
to be affected predominantly by shear and compression ahead of the tool along the cutting direction (Figures 4(a) and (b)). In the case of the (101)

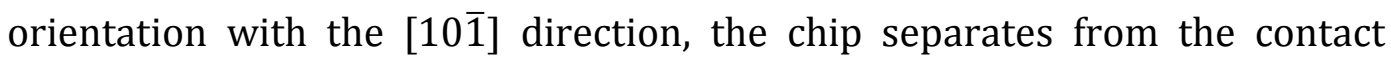
region on the rake face rapidly (Figures 4 (c) and (d)). A comparison of chip morphologies after machining shows similarity in the type of chip formed for copper and aluminium. It can be seen that the nature of the deformation ahead of the tool, the motion of the dislocations, the subsurface deformation, and side flow of the work piece material depend strongly on the orientation and direction of cutting.

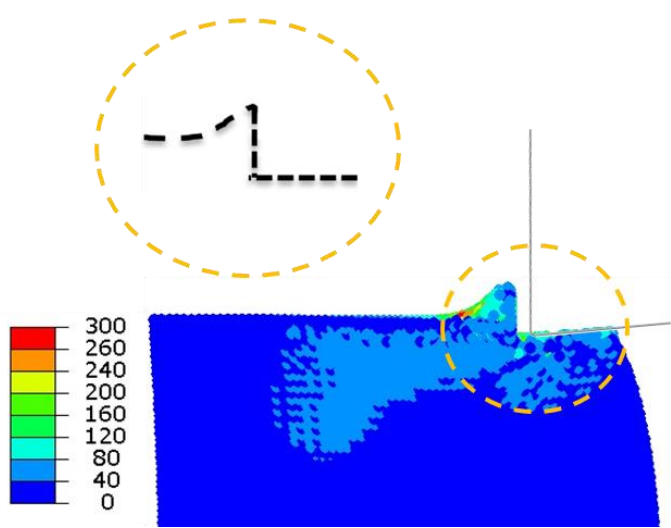

(a) aluminium machining at (100)[100]

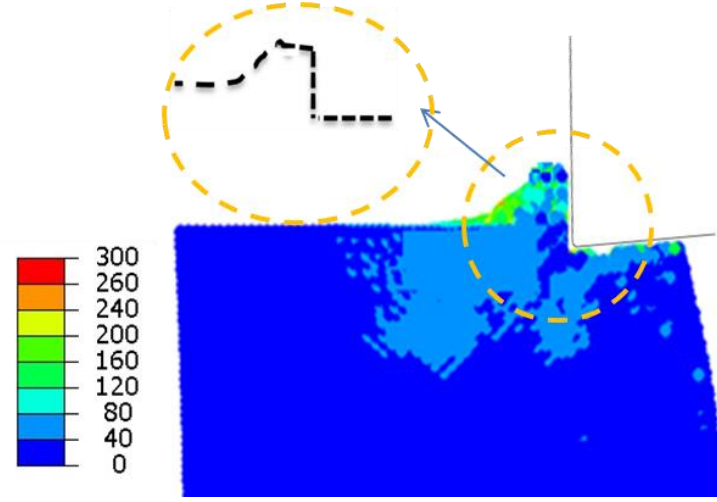

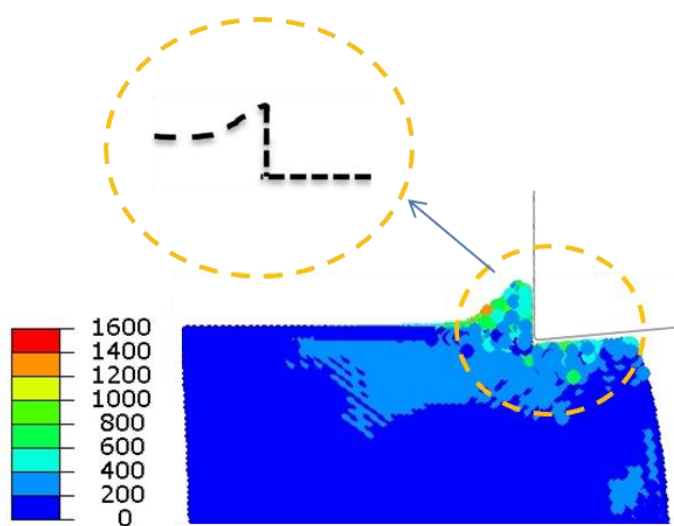

(b) copper machining at (100)[100]

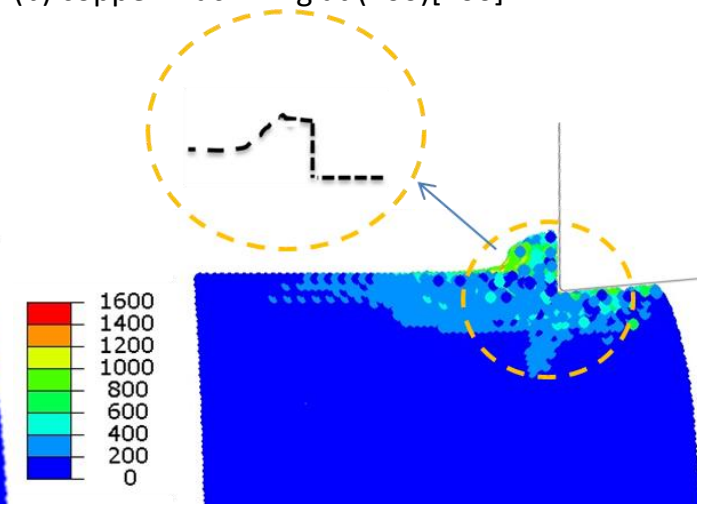

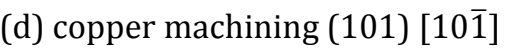

(c) aluminium machining at (101) [10 $\overline{1}]$

Figure 4: Chip formation pattern and distribution of von Mises stresses (in MPa) in machined single crystals

The magnitude of cutting forces, on the other hand, has a stronger dependence on the material parameters. The level of von Mises stresses in machining of copper presented in Figure 4 is around 5 times higher than that for aluminium. 


\section{Concluding remarks}

The mechanism of deformation in single-crystal machining is complex. The importance to account for grain orientation and direction of machining in assessment of the overall cutting forces was demonstrated together with propensity of the workpiece material to generate slips when its grains undergo rotations. Comparing the cutting force in aluminium and copper showed that though the magnitude of the cutting force is different in two materials cut under the same conditions the character of variation of the cutting force for different orientation for both materials was similar.

\section{Acknowledgement}

The authors are grateful to the EPSRC UK (grant EP/K028316/1) for financial support of these studies.

\section{References}

[1] Okazaki, Y., Mishima, N., Ashida, K.: Microfactory: concept, history, and developments. J. of man. sci. and eng. 126 (4) 837-844 (2004).

[2] Masuzawa, T.: State of the art of micromachining. CIRP ann.- manu. tech. 49 (2) 473-488. (2000)

[3] Zahedi, S.A., Demiral, M., Roy, A., Silberschmidt, V.V.: FE/SPH modelling of orthogonal micro-machining of f.c.c. single crystal. Comp. mat. sci. 78 104-109 (2013)

[4] Zahedi, S.A., Roy, A., Silberschmidt, V.V.: Modeling of micro-machining single-crystal f.c.c. metals. Proc. CIRP 8 346-350 (2013)

[5] Sato, M., Kato, Y., Aoki, S., Ikoma, A.: Effects of crystal orientation on the cutting mechanism of the aluminum single crystal: 2nd Report: On the (111) plane and the (112) end cutting. Bull. of JSME 26 (215) 890-896 (1983)

[6] Lee, W., Yang, W.: Methodology and applications of mesoplasticity in manufacturing sciences, Int. J. of mech. sci. 35(12) 1079-1095 (1993)

[7] Lee, W.B., To, S., Sze, Y.K, Cheung, C.F.: Effect of material anisotropy on shear angle prediction in metal cutting- a meso-plasticity approach. Int. j. of mech. sci.45 (10) 1739-1749 (2003)

[8] Shirakashi, T., Yoshino, M., Kurashima, H.: Study on cutting mechanism of single crystal based on simple shear plane model, Int. j. jap. pre. Eng. 25 (2) 96-97 (1991).

[9] Lawson, B.L., Kota, N., Ozdoganlar, O.B.: Effects of crystallographic anistropy on orthogonal micromachining of single-crystal aluminium. Tran. of the ASME-B-J. of man. sci. and engine. 130 (3) $316-319$ (2008)

[10] Chunhui, Ji., Shi, J., Liub, Z., Wang, Y., Comparison of tool-chip stress distributions in nanomachining of mono-crystalline silicon and copper, Int. j. of mech. sci. 77 30-39 (2013)

[11] Komanduri, R., Chandrasekaran, N., Raff, L.: MD simulation of exit failure in nanometric cutting, Mat. sci. and eng. A, 311(1) 1-12 (2001).

[12] Cai, M.B., Li, XP., Rahman, M.: Study of the mechanism of nano-scale ductile mode cutting of silicon using molecular dynamics simulation. Inte. j. of mach. tools and man. 4755-480. (2007)

[13] Hutchinson, J.: Bounds and self-consistent estimates for creep of polycrystalline materials, proc. of the Roy. Soc. of Lon. A. Math. Phy. \& eng.sci. 348 101-127 (1976).

[14] Peirce, D., Asaro, R.J., Needleman, A.: An analysis of nonuniform and localized deformation in crystalline solids, Acta. metal. 30 1087-1093 (1982)

[15] Huang, Y.: A user-material Subroutine incorporating single crystal plasticity in the ABAQUS finite element program, Harvard Uni. (1991) 
[16] Kysar, J.: Addendum to a user-material subroutine incorporating single crystal plasticity in the ABAQUS finite element program, Mech. rep. 178 (1997)

[17] Zahedi, S.A., Crystal plasticity modelling of machining, $\mathrm{PhD}$ thesis, Loughborough Univeristy (2014)

[18] Zahedi, S.A., Demiral, M., Roy, A., Babitsky V.I, Silberschmidt, V.V. Indentation in fcc single crystals, Solid sta. phen. 188 219-225

[19] Demiral, M., Enhanced gradient crystal-plasticity study of size effects in b.c.c. metal, $\mathrm{PhD}$ thesis (2012)

[20] Wang, Y., Raabe, D., Klüber, C., Roters, F.: Orientation dependence of nanoindentation pileup patterns and of nanoindentation microtextures in copper single crystals, Acta mat. 52 (8) 22292238 (2004)

[21] Groh, S., Marin, E., Horstemeyer, M., Zbib, H.: Multiscale modelling of the plasticity in an aluminum single crystal. Int. J. of plas. 25(8) 1456-1473 (2009) 